\title{
Etiologie de 350 cas de stérilité masculine. Effet de divers traitements sur la qualité du sperme et analyse de leur rôle dans la survenue de $\mathbf{1 0 0}$ grossesses
}

\author{
R.C. Martin-Du Pan, A.Campana
}

Clinique de Stérilité et d'Endocrinologie de la Reproduction, Hôpital Cantonal Universitaire de Genève

\section{RESUME}

Des facteurs étiologiques de stérilité masculine et l'effet de différents traitements sur la qualité du sperme ont été évalués chez 350 patients consultant pour une stérilité primaire ou secondaire de plus d'un an. Des facteurs d'environnement ont été mis en évidence dans $12 \%$ des cas, des maladies acquises telles que prostatite, varicocèle ou auto-immunité dans $36.8 \%$, une insuffisance testiculaire dans $23 \%$, une azoospermie obstructive dans $7,4 \%$ et une endocrinopathie dans $1,4 \%$ des cas. Dix-neuf $\%$ des cas étaient idiopathiques.

Un traitement conventionnel a pu être proposé dans $50 \%$ des cas et évalué chez 101 patients. Une normalisation du sperme (définie par un NTSM de plus de 16 millions) a été observée dans le \% de cas suivants : $80 \%$ après hormonothérapie, $41 \%$ après antibiothérapie pour prostatite, $37 \%$ après cure de varicocèle et $30 \%$ après un traitement anti-inflammatoire de diclofénac.

Sur 100 grossesses, $39 \%$ sont survenues spontanément ou après traitement inducteur d'ovulation, $32 \%$ à la suite de traitement andrologique conventionnel et $26 \%$ à la suite de procréation médicalement assistée telle que fécondation in vitro clas. sique (FIV), injection intracytoplasmique de spermatozoïdes (ICSI) ou insémination intra-utérine (IIU). L'efficacité relative de ces divers traitements en fonction du NTSM et l'utilité de l'investigation de l'homme infertile sont discutées. Les risques encourus par la femme lors de traitement par ICSI sont soulignés.

Mots-clés : stérilité masculine, traitement médicamenteux, fécondation in vitro, étiologie.

\section{INTRODUCTION}

On admet que $15 \%$ des couples présentent un problème d'infertilité et que l'homme en est responsable dans près de la moitié des cas [1]. Les traitements de la stérilité masculine ont connu récemment des succès spectaculaires grâce aux techniques de fécondation in vitro, en particulier l'injection intra-ovocytaire de spermatozoïdes (ICSI) [2]. Grâce à l'ICSI on peut obtenir des grossesses en cas de stérilité due à des altérations sévères du sperme [3]. Du fait de ces progrès l'évaluation et le traitement de la stérilité masculine reposent de plus en plus sur l'examen et la manipulation du sperme par le gynécologue et le biologiste. On tend à négliger la recherche de facteurs étiologiques à l'origine des anomalies séminales [4]. Certains estiment que la recherche de tels facteurs est souvent inutile puisque dans plus de $50 \%$ des cas il s'agit de stérilité d'origine 
inconnue ("idiopathique") [5]. De plus même si l'on trouve une cause telle qu'une varicocèle ou une prostatite, il n'est pas prouvé que le traitement de ces affections augmente les chances de grossesse [5].

Est-il donc utile d'examiner l'homme infertile et de rechercher des causes de stérilité masculine ? Y-a-t'il encore une place pour les traitements conventionnels de la stérilité masculine (en dehors des techniques de procréation médicalement assistée (PMA)) ? Permettent-ils d'améliorer la qualité du sperme, d'augmenter les chances de grossesse?

Le but de ce travail est de répondre à ces questions à partir de l'étude de 350 hommes infertiles qui ont été adressés par les gynécologues de leur femme en raison d'une stérilité primaire ou secondaire de plus d'une année dont on suspectait une origine masculine vu l'absence de cause évidente de stérilité féminine. Toutes les femmes avaient au moins une trompe perméable à l'hystérosalpingographie. En revanche certaines d'entre elles présentaient des cycles anovulatoires, qui ont nécessité un traitement de clomifène (Clomid).

\section{Il s'agissait :}

- d'évaluer la prévalence des différents facteurs étiologiques de stérilité masculine dans cette population ;

- d'établir une corrélation entre facteurs étiologiques et sévérité des altérations séminales ;

- d'observer l'effet de différents traitements conventionnels sur la qualité du sperme ;

- d'analyser les traitements ayant abouti à 100 grossesses, en fonction des diverses causes de stérilité masculine ;

- de discuter l'efficacité relative et les risques des divers traitements selon les données récentes de la littérature.

\section{MATERIEL ET METHODE}

\section{Patients}

Il s'agissait de 350 patients ayant consulté entre 1985 et 1995 en raison d'une stérilité primaire de plus d'une année $(73 \%)$ ou secondaire de plus de 2 ans $(27 \%)$. Ils étaient porteurs d'une ou plusieurs anomalies du sperme. Leur âge variait entre 22 et 61 ans (31 ans en moyenne). La durée moyenne de l'infertilité était de $24+8$ mois (+ SD). L'anamnèse et l'examen physique des patients ont été réalisés en fonction des directives de l'OMS par le même médecin (MDP) [6].

\section{Spermogramme}

Deux échantillons de sperme au moins ont été analysés après 3-4 jours d'abstinence, à 2 mois d'intervalle. L'analyse du sperme a été effectuée par une méthode computérisée permettant une mesure objective de la concentration et de la mobilité du sperme grâce à un système de photographies à expositions multiples (Spermamat, Softool, SA, Suisse), [7].

La morphologie du sperme a été évaluée au microscope à contraste de phase après coloration de Papanicolaou. Différents paramètres du sperme ont été définis selon les directives de l'OMS (1987) [8] : volume, concentration, pourcentage de spermatozoïdes mobiles et de morphologie normale. En outre le nombre total de spermatozoïdes mobiles par éjaculat (NTSM) a été calculé pour chaque patient (NTSM= nombre total de spermatozoïdes /éjaculat $\mathrm{x} \%$ de formes mobiles). On considère selon les critères de l'OMS que le sperme est anormal si le nombre total de spermatozoïdes par éjaculat est de moins de 40 millions, si le TSM est de moins de $16(=40 \times 40 \%)$ millions/ éjaculat ou s'il y a moins de $40 \%$ de formes normales. Les anticorps antispermatozoïdes ont été mesurés en utilisant le test d'immunobilles dans le sperme (immunobilles de lapin antiIgG, IgA humaines, 
IRVINE Scientific Santa Ana CA) en cas de NTSM de moins de 10 millions ou en cas d'asthénospermie avec moins de $20 \%$ de formes mobiles.

\section{Echographie testiculaire}

Une échographie a été pratiquée en utilisant une sonde de $5 \mathrm{MHz}$ d'un appareil Hitachy Eub 240 en cas de palpation ou de volume anormal des testicules afin de détecter épididymite, spermatocèle, tumeur intrascrotale etc. Une échographie a aussi été pratiquée en cas de varicocèle, diagnostiquée en présence d'au moins deux veines spermatiques dilatées de plus de $2 \mathrm{~mm}$, d'une hyperthermie scrotale et d'un reflux continu au doppler en valsalva. Les cas de varicoèle sub-cliniques n'ont pas été pris en compte.

\section{Examens sanguins}

L'hormone folliculostimulante (FSH) et l'hormone lutéinisante (LH) ont été mesurées dans le sérum par une méthode immunoenzymatique utilisant 2 anticorps monoclonaux (Stratus ; Baxter Düdingen. Limite de détection $0,5 \mathrm{miU} / \mathrm{ml}$ ). La LH, la FSH et la testostérone n'ont été dosées qu'en cas de concentration du sperme de moins de 10 millions $/ \mathrm{ml}$. La prolactine n'a été mesurée qu'en cas d'impuissance. Le diagnostic d'abus d'alcool (plus de $60 \mathrm{~g} / \mathrm{j}$ ) n'a été retenu qu'en cas de perturbation de la biologie hépatique.

\section{Biopsie testiculaire}

Une biopsie a été réalisée en cas d'azoospermie avec volume testiculaire normal et taux normal de FSH ou en cas d'exploration testiculaire pour une azoospermie obstructive si aucun spermatozoïde n'était présent dans les épididymes.

\section{Classification étiologique de la stérilité}

La classification était basée d'une part sur des facteurs étiologiques spécifiques toxiques, infectieux, immunologiques, vasculaires, mécaniques ou tumoraux, d'autre part sur les possibilités de traitement $[1,5]$. De façon schématique les causes acquises de stérilité bénéficient de traitements conventionnels, tandis qu'en cas d'atteinte de l'épithélium germinatif ou d'atteintes congénitales on recourt aux techniques de PMA telles que l'ICSI) [9]. On définit comme idiopathiques les cas où l'on observe des altérations du sperme mais où l'examen physique, les dosages sanguins et l'anamnèse ne révèlent aucune anomalie.

On n'a retenu qu'un seul diagnostic par patient. Lorsque plus d'un diagnostic était présent on a retenu celui qui semblait le plus délétère sur la fonction testiculaire (par ex., une cryptorchidie par rapport à une varicocèle, des facteurs immunologiques par rapport à une prostatite, des facteurs hormonaux par rapport à une dysfonction sexuelle etc.).

\section{Traitements}

\section{a) traitements conventionnels}

En cas de prostatite, définie d'après les critères de Comhaire et coll. [10], on administra de la ciprofloxacin $(2 \times 500 \mathrm{mg} / \mathrm{j})$ pendant 3 semaines aux 2 partenaires (en cas de cervicite associée) en recommandant l'usage de condoms. En cas de leucospermie persistante (plus de 1 million de leucocytes $/ \mathrm{ml}$ ) une deuxième cure de ciprofloxacin a été prescrite. Un traitement de doxycycline $(200 \mathrm{mg} / \mathrm{j}$. pendant $8 \mathrm{j}$. a été prescrit au couple en présence de chlamydia dans les cellules uréthrales détectée par immunofluorescence (Chlamydia direct IF, Biomérieux).

En cas d'oligospermie sévère (NTSM 3) dans 2 analyses consécutives de sperme et d'un taux normal de FSH (4 UI/l), le tamoxifène $(2 \times 10 \mathrm{mg} / \mathrm{j})$ a été prescrit pendant 10 semaines si le taux sérique de FSH avait augmenté au moins de $50 \%$ après une semaine de traitement. 
En cas d'azoospermie ou d'oligospermie très sévère (NTSM 1) avec un taux normal de FSH, un anti-inflammatoire,le diclofénac, $(100 \mathrm{mg} / \mathrm{j})$ a été prescrit pendant 3 semaines.

En cas de varicocèle, une opération a été proposée selon les critères de Nieschlag et coll [11]. L'opération consistait en la ligature haute des veines spermatiques selon la technique de Palomo. Depuis 1992, l'opération a été pratiquée par laparoscopie. La plupart des opérations ont été pratiquées par le même urologue.

\section{b) techniques de reproduction assistée}

Elles ont été pratiquées par les différents gynécologues qui nous ont adressé les conjoints de leurs patientes. Parmi eux quatre gynécologues, utilisant les compétences du même biologiste, ont procédé aux techniques de fécondation in vitro (FIV).

- une insémination intra-utérine (IIU) avec le sperme sélectionné par la méthode du swim-up, décrite par Arny et Quagliarello [12] a été effectuée en cas d'oligospermie idiopathique ou d'échec des traitements conventionnels si le TSM était 3.

- la fécondation in vitro classique (FIV c) selon une technique décrite précédemment [13] a été effectuée en cas de stérilité idiopathique de plus de 3 ans ou d'échec de traitements conventionnels ou d'IIU et en cas de NTSM situé entre 1 et 3. En principe la FIV était réalisée en raison de stérilité masculine et non de stérilité tubaire une trompe au moins étant perméable.

- l'ICSI a été utilisé depuis 1995 seulement, en cas d'échec de FIV ou de NTMS de moins de 1 selon une technique décrite ailleurs [14] et adaptée de la technique initialement utilisée par Palermo et coll.

\section{c) Traitement inducteur d'ovulation (clomifêne)}

En cas de cycles anovulatoires un traitement de clomifêne (Clomid) a été instauré chez les femmes des hommes infertiles. En cas de grossesse et en l'absence de traitement de la stérilté masculine par PMA (IIU) on considère le facteur masculin comme peu significatif et ces cas ont été regroupés avec ceux de grossesse spontanée (cf figure 2).

\section{Grossesses}

Seules les grossesses à terme ont été enregistrées. En cas de grossesse multiples ou de la survenue de plusieurs grossesses dans un couple, une seule grossesse a été comptabilisée. On a défini comme grossesse spontanée (ou indépendante du traitement) toute grossesse survenue plus de 6 mois après un traitement médicamenteux ou chirurgical de stérilité masculine. Autrement dit seules les grossesses survenant dans les 6 mois suivant la fin d'un traitement ont été considérées comme résultant d'un traitement spécifique (conventionnel ou par PMA) (cf figure 3). Arbitrairement l'étude a été stoppée après la survenue de la 100è grossesse.

\section{Analyse statistique}

Un test de student réalisé sur le logarithme des valeurs de NTSM a été utilisé pour déterminer si des modifications du NTSM après traitement étaient significatives (paired test).

\section{RESULTATS}

\section{Facteurs étiologiques}

Ils sont résumés dans la Figure 1 . Ils ont été identifiés dans $80 \%$ des cas. Les facteurs d'environnement (drogues et médicaments, alcool, exposition à la chaleur) représentent $12 \%$ des cas, les maladies acquises (varicocèle, prostatite, chlamydia, anticorps antispermatozoïdes, torsion ou cancer testiculaire) $36.8 \%$, les insuffisances testiculaires (cryptorchidie, hypospermatogenèse, azoospermie sécrétoire, arrêt de la spermatogenèse) $23 \%$, les ano- 


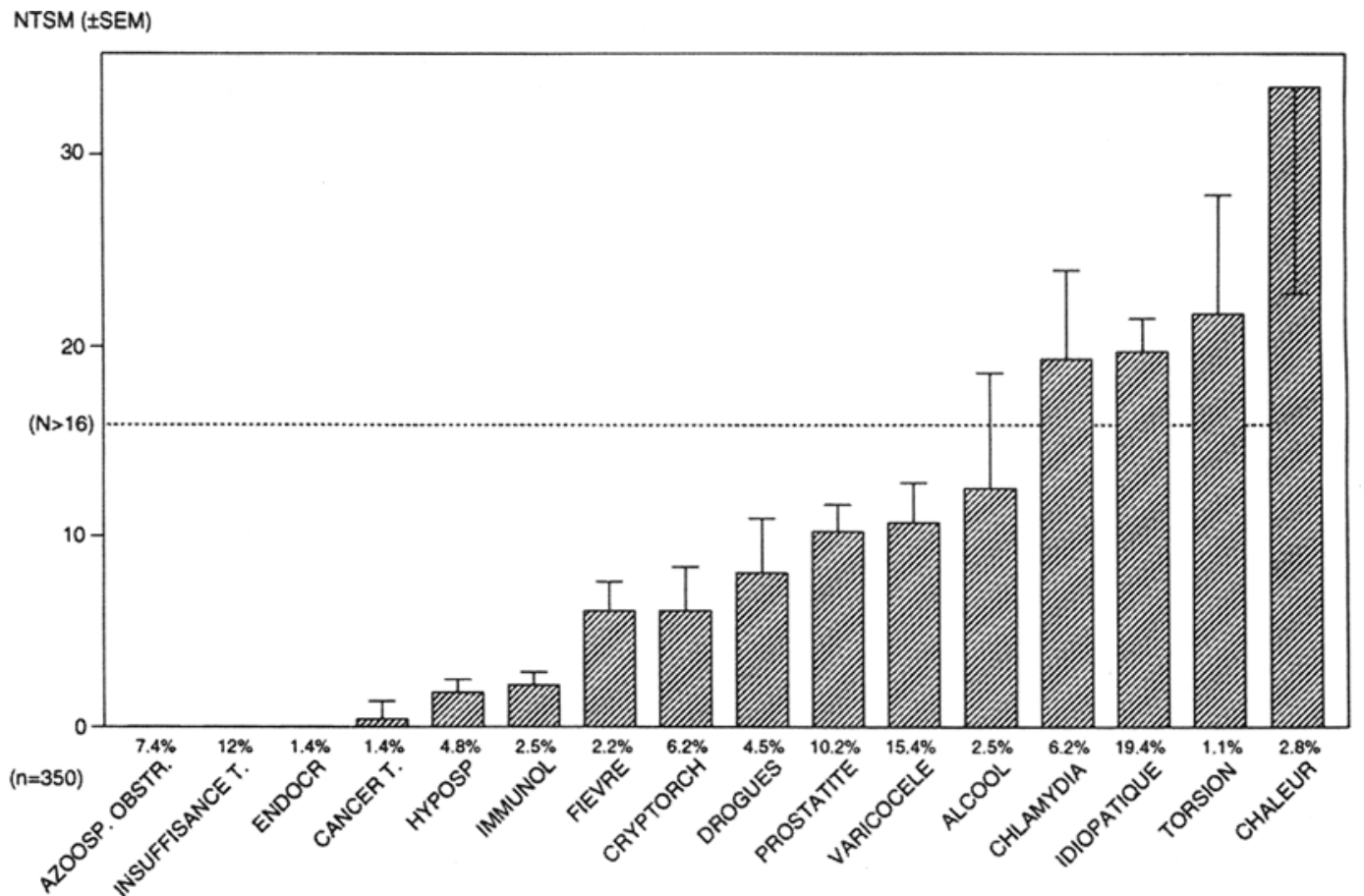

Figure 1 : Prévalence (\%) de diférentes catégories étiologiques de stérilité masculine en fonction du nombre total de spermatozoïdes mobiles (NTSM).

malies de transport du sperme $7,4 \%$, les dysfonctions sexuelles et les endocrinopathies $1,4 \%$. Dans $19,4 \%$ des cas l'étiologie était idiopathique. L'analyse du sperme, évaluée chez 350 patients par le nombre total de spermatozoïdes mobiles par éjaculat (NTSM), est résumée dans la Figure 1. Un NTSM de moins de 5 millions a été mesuré en cas d'endocrinopathie, d'autoimmunité, de cancer testiculaire et en cas d'insuffisance testiculaire congénitale ou acquise. Un NTSM situé entre 5 et 15 a été mesuré en cas de prostatite, de varicocèle et de cryptorchidie. Un NTSM de plus de 15 millions a été observé en cas d'infection à chlamydia, d'exposition professionnelle à la chaleur ou de dysfonction sexuelle.

Chez 20 patients exclus de l'étude en raison d'un spermogramme normal, le NTSM moyen était de $89+16$ (SEM) (non figuré).

\section{Effet de traitements conventionnels sur la qualité du sperme}

L'effet de différents traitements sur le NTSM est résumé dans la figure 2. Il n'a pu être évalué que chez 101 patients, soit parce qu'ils n'ont pas voulu redonner du sperme après le traitement, soit parce que leur femme était enceinte ou qu'ils ont été perdus de vue. Chez 20 patients présentant une azoospermie ou une oligospermie sévère (moins de 1 million de spermatozoïdes/ ml) l'administration de diclofénac (Voltarène) a permis d'induire l'apparition de plus de 15 millions de $\mathrm{sp} / \mathrm{ml}$ chez 6 d'entre eux. Dans 5 cas d'azoospermie due à une insuffisance hypophysaire ( 3 cas) et à des prolactinomes ( 2 cas) l'administration de gonadotrophines de la ménopause (HMG) ou de bromocriptine a permis de normaliser le sperme dans 4 cas avec l'obtention de 3 grossesses. 


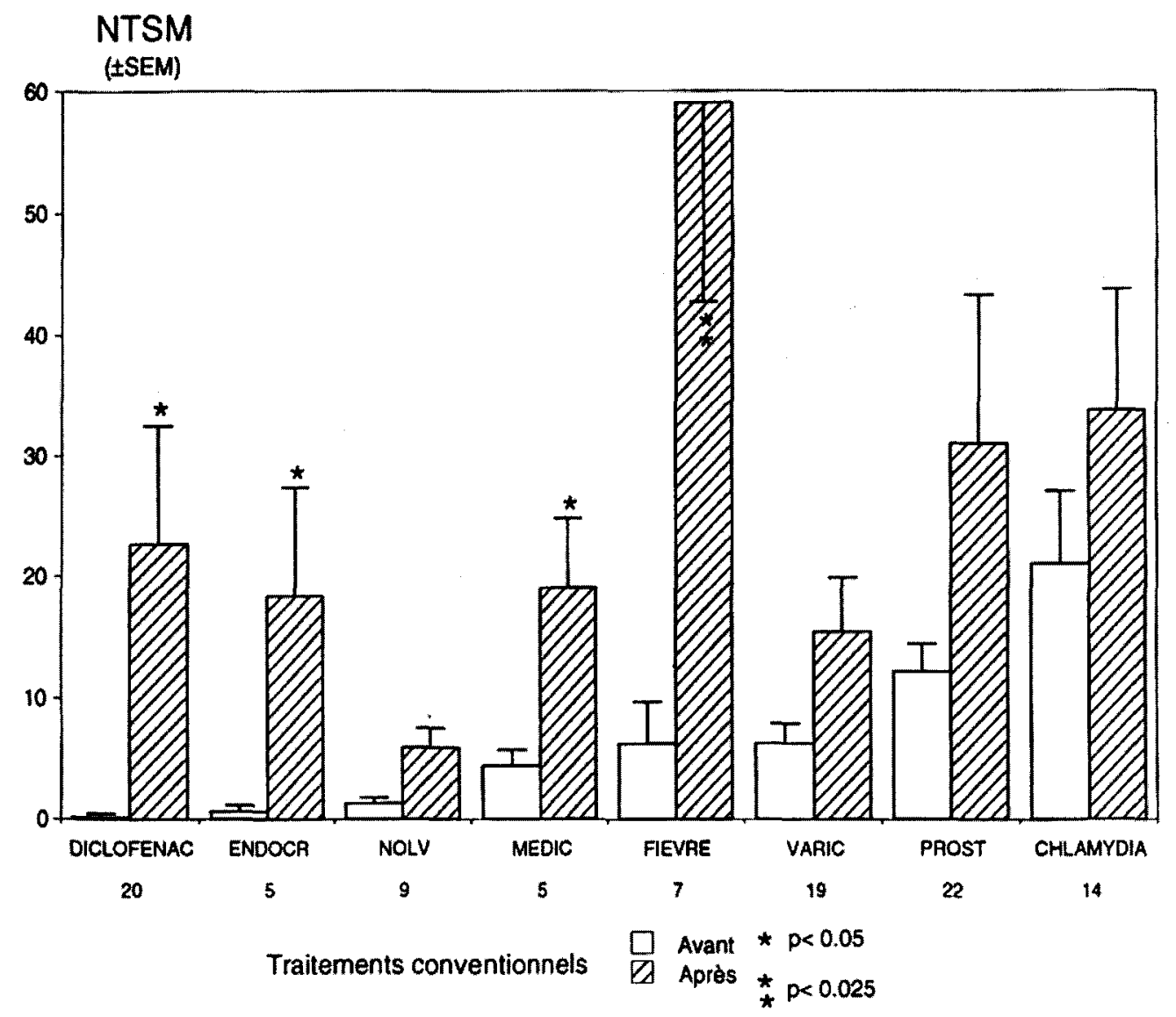

Figure 2 : Effet de différents traitements conventionnels sur le nombre total de spermatozoïdes mobiles (NTSM).

L'administration de tamoxifène (Nolvadex) a permis une augmentation du NTSM chez $4 / 9$ patients, de $1,3+0,5(\mathrm{SD})$, à $7,5+$ 1,7. avec la survenue d'une grossesse. Chez les 5 autres patients le NTSM n'a pas augmenté. Le \% moyen de formes normales chez les 9 patients était de $12,2 \%+5,9$ (SD).

L'effet résultant de l'arrêt de médicaments ou de drogues ayant un effet délétère sur le sperme a été observé chez 5 patients. Deux patients rendus azoospermiques par la prise d'androgènes ont normalisé leur sperme 9 mois après l'arrêt des stéroïdes avec l'obtention de 2 grossesses. Dans 3 autres cas on note une augmentation de plus de $100 \%$ du NTSM après remplacement de phénytoïne par de la cabamazépine ( 1 cas), diminution de la dose de méthadone ( 1 cas) et arrêt du hashish ( 1 cas). Trois grossesses sont survenues après arrêt d'une drogue ou d'un médicament.

Dans 7 cas de NTSM abaissé (en moyenne $6,3+8,9(\mathrm{SD}))$ suite à un épisode fébrile, un contrôle du sperme 3 mois plus tard a montré une amélioration spontanée du sperme qui a augmenté en moyenne à $59+$ 16.

Dans 19 cas où l'on a pu analyser le sperme après une opération de varicocèle, on a noté une augmentation non significative $(p>0.05)$ du NTSM qui passe de $6,2+1,6$ (SD), en moyenne à $15,4+4,4$. Aucune amélioration du NTSM n'a eu lieu dans 6 
cas dont le NTSM était $<2$, alors qu'on note une augmentation de plus de $300 \%$ dans 7 cas où le NTSM était situé entre 2 et 6 . Neuf grossesses sont survenues parmi les 29 patients opérés de leur varicocèle $(31 \%)$.

Une amélioration non significative ( $\mathrm{p}>$ 0.05) du NTSM a été observée après traitement antibiotique en cas de prostatite, qui augmente de plus de $200 \%$ dans 10 des 22 cas traités. Le NTSM augmente en moyenne pour l'ensemble des 22 cas de $12,2+2,3$ à $31+12,3$ (ns).

Dans les cas de chlamydia, une augmentation du NTSM a été observée dans 8 des 14 cas traités mais elle n'était pas significative pour l'ensemble du groupe. Dans l'ensemble on a obtenu 7 grossesses parmi les 36 patients traités pour prostatite $(19 \%)$ et 6 parmi les 22 traités pour chlamydia (27\%).

\section{Effet des différents traitements sur les grossesses}

La figure 3 résume les différents traitements ayant abouti à la survenue de 100 grossesses en fonction des valeurs de NTSM. Il ne s'agit pas forcément des mêmes sujets que dans la figure 2 puisque certaines techniques de PMA ont été appliquées sans traitement andrologique préalable ( ou sans que le résultat de ce dernier soit disponible). Lors de la survenue de grossesses spontanées ( $24 \%$ des cas) et en l'absence de traitement spécifique d'éventuels facteurs masculins de stérilité, on a observé une valeur moyenne de NTSM de $30+9$ (SD). Le NTSM était en moyenne de $22+4,4$ chez les maris de femmes devenues enceintes à la suite d'un traitement de clomifène, en raison de cycles anovulatoires (14\% des cas). Ces valeurs de NTSM ne sont pas significativement différentes de celles observées dans le groupe traité par

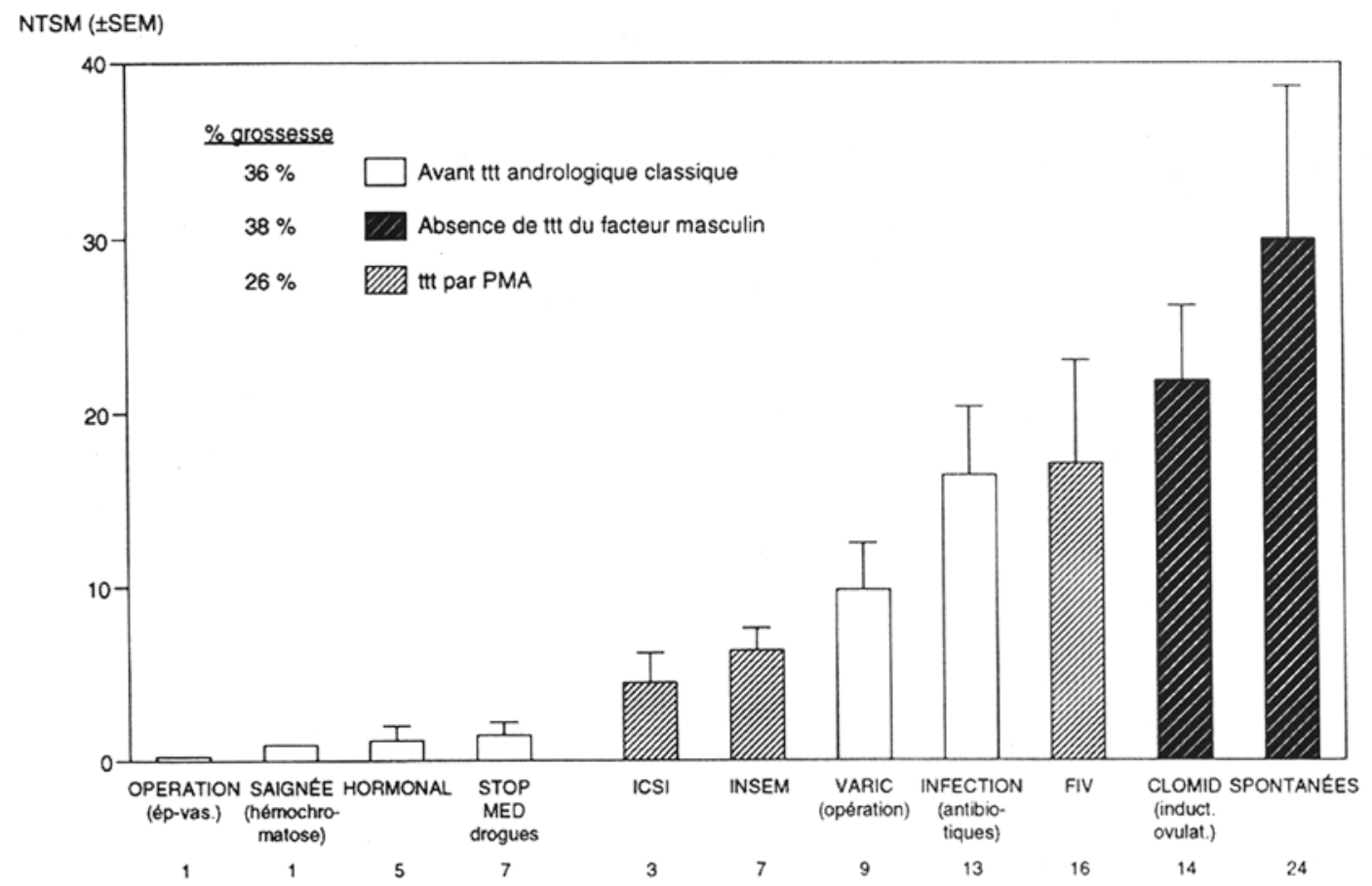

Figure 3 : Effet de différents traitements sur la survenue de 100 grossesses en relation avec le nombre total de spermatozoïdes mobiles (NTSM). 
fécondation in vitro classique (NTMS $=17,1$ +6) (13\% des cas) ou dans le groupe traité par antibiotique pour une prostatite ou une infection à chlamydia (NTSM $=16,4+4)$ (8\% des cas). En revanche le NTSM était significativement plus bas ( $p$ 0.05) dans les cas traités par insémination intra-utérine ou par ICSI. Une grossesse est survenue à la suite de saignées en raison d'une hémochromatose, une autre à la suite d'une épididymovasostomie en raison d'une azoospermie obstructive.

Nous avons connaissance de 2 malformations majeures parmi ces 100 grossesses $(2 \%)$ : un cas d'anencéphalie avec décès peu après la naissance, survenu après FIV ; un cas de malformation digestive avec éventration corrigée chirurgicalement après la naissance chez un enfant par ailleurs normal. La grossesse était survenue spontanément après 3 tentatives infructueuses de FIV.

\section{DISCUSSION}

\section{Facteurs étiologiques : données épi- démiologiques}

La recherche attentive de facteurs étiologiques nous a permis de trouver des anomalies biologiques (infections, auto-immunité) hormonales, vasculaires ou tumorales ou des facteurs toxiques d'environnement dans près de $80 \%$ des cas de stérilité masculine. Ces différents facteurs ont été revus en détail ailleurs [15]. L'intérêt de notre étude est qu'elle évite certains biais de sélection contrairement à différents centres (cliniques urologiques) qui recrutent un nombre élevé de cas de varicocèle ou un nombre élevé de maladies endocriniennes en raison d'un intérêt particulier pour ces maladies $[16,17]$.

\section{Utilité diagnostique et valeur pro- nostique du NTSM}

Plusieurs travaux ayant remis en question la valeur clinique de la morphologie dans la fécondation in vivo [18, 19], nous nous sommes intéressés surtout au NTSM. Ce dernier était significativement plus bas dans des causes bien établies d'infertilité (hormonales ou autoimmunes) que dans des causes plus controversées (varicocèle,prostatite) [15]. Une étude prospective portant sur 1055 patients infertiles a montré une diminution de la fertilité pour un NTSM de moins de 25 millions [20]. Après vasovasostomie $10 \%$ des grossesses seulement ont été obtenues chez des patients ayant un NTSM de moins de 10 millions et aucune grossesse n'a été obtenue en cas de NTSM de moins de 3 [21]. Il en est de même en cas d'IIU [22]. En cas de FIV aucune fécondation n'a lieu si le NTSM après swim-up est situé en dessous de valeurs variant entre 0,5 à 3 millions, selon les techniques de FIV $[23,24]$. Le NTSM est donc utile et pour évaluer le pronostic et pour orienter le choix d'une thérapie.

3. Effets de divers traitements sur le NTSM et sur le taux de grossesse selon l'étiologie de la stérilité masculine

\section{a) Problèmes méthodologiques}

$\mathrm{Vu}$ la variabilité spontanée du sperme et le phénomène de régression vers la moyenne [25], il est très difficile de démontrer l'efficacité d'un traitement en raison d'une erreur statistique de type 2. Le fait d'avoir au moins 2 spermogrammes de départ (avant le traitement), comme dans notre étude, permet toutefois d'atténuer ce phénomène.

\section{b) Prostatite et infection à Chlamydia}

Une augmentation du sperme a été notée dans près de $50 \%$ de nos cas de prostatite et de chlamydia après antibiothérapie mais elle n'était pas significative. Le rôle des germes infectieux dans la prostatite et le rôle de cette dernière dans l'infertilité sont controversés [26]. Deux études contrôlées ont montré que la leucospermie se normalisait souvent spontanément [10, 27]. Cependant elle tend à récidiver et seule l'antibio- 
thérapie associée à des éjaculations fréquentes permet d'obtenir des effets durables [28].

\section{c) Opération de varicocèle}

Bien que de nombreuses études non contrôlées -dont la nôtre- aient montré une amélioration du sperme après cure chirurgicale, le rôle de la varicocèle dans l'infertilité masculine reste controversé $[11,29,30]$. Dans notre étude l'opération n'a amélioré le sperme d'aucun patient dont le NTSM était $<2$. Mais si le NTSM initial est de plus de 15, il est peu probable que la varicocèle soit responsable de l'infertilité et la principale indication opératoire serait d'éviter une détérioration ultérieure de la fonction testiculaire.

\section{d) Traitement par le tamoxifène}

En cas de stérilité d'origine idiopathique, une méta-analyse a montré que les antioestrogènes, en particulier le tamoxifene pourrait être supérieur au placebo [31]. Dans notre étude de patients ayant une oligospermie sévère seul 4 cas sur 9 ont répondu au traitement mais il n'y a eu qu'une grossesse peutêtre en raison de la tératospermie, souvent associée à ces cas d'oligospermie sévère.

\section{e) Traitement par le diclofénac}

Des études non contrôlées ont montré un effet bénéfique des anti-inflammatoires en cas d'oligospermie [32]. Cependant l'amélioration rapide (3 semaines) obtenue chez nos patients suggère que le diclofenac a agi sur une possible composante obstructive. Certains cas d'oligospermie sévère considérés comme idiopathiques pourraient être dûs en fait à une occlusion unilatérale ou incomplète des épididymes survenue à la suite d'une infection, ce que Schoysman a appelé l'"oligospermie épididymaire" [33-36].

\section{Techniques de reproduction assistée \\ a) Insémination intra-utérine}

L'insémination intra-utérine avec le sperme activé par la méthode de swim-up a permis d'obtenir 7 grossesses dans des cas où le TMS était assez bas (TMS moyen $=6,3+$ 1,1 (+ SEM). On admet qu'il faut au moins 3 millions de spermatozoïdes mobiles après swim-up pour tenter une IIU, avec un taux de grossesse de $5,6 \%$ par cycles après IIU contre $1,3 \%$ après rapports sexuels seulement [22, 37-39]. Ce taux peut être aug. menté en couplant l'IUU à un traitement inducteur d'ovulation $[39,40]$.

\section{b) Fécondation in vitro classique (FTV c)}

$16 \%$ des grossesses sont survenues à la suite d'un traitement de FIV. Le NTSM (17 +6) n'était pas significativement différent dans le groupe FIV que dans les cas de grossesses spontanées $(30+9)$ ce qu'on peut expliquer par la présence dans plusieurs cas d'une tératospermie justifiant la FIV en présence d'un NTSM peu altéré.

Le recours à la FIV n'est possible que s'il y a au moins 500.000 spermatozoïdes mobiles après sélection et activation du sperme par swim-up ou minipercoll [24]. Hinting et coll. [42] ont montré que la FIV permettait de doubler le taux de grossesse par rapport aux traitements conventionnels de la stérilité masculine ( $8 \%$ par cycle contre $4 \%$ ). Crosignani et Walters [41] ont observé un taux de grossesse moyen de $21 \%$ par cycle après IIU ou FIV contre $6,8 \%$ après un seul traitement inducteur d'ovulation.

En l'absence de fécondation des ovocytes in vitro en raison d'une OAT extrême (moins de 500.000 spermatozoïdes mobiles) ou d'autres facteurs (immunologiques etc), ou en cas d'échecs répétés de FIV, on recourt actuellement à l'ICSI [2].

\section{c) Injection intra-ovocytaire de sperma- tozoïde (ICSI)}

Cette méthode n'étant disponible à Genève que depuis 1994 [14], nous n'avons obtenu dans cette étude que 3 grossesses avec cette technique développée en 1992 par Palermo et coll. [2]. 
L'ICSI a révolutionné le traitement de la stérilité masculine puisqu'elle a permis de réaliser une fécondation des ovocytes en cas d'échec de la FIV classique et surtout en cas d'altération sévère de tous les paramètres du sperme [3]. Des grossesses ont été obtenues en cas d'azoospermie obstructive et même en cas d'azoospermie sécrétoire [9]. Bien que l'on n'ait pas mis en évidence davantage de malformations chez les enfants nés par ICSI que dans la population générale ou après FIV $c[2,36]$, on ne devrait recourir à cette technique qu'en dernière extrémité. En effet, outre le risque d'infertilité pour la descendance [44], la FIV et l'ICSI imposent à la femme des risques non négligeables, alors qu'elle n'a elle-même parfois aucun problème d'infertilité [45]. Elles peuvent entraîner des complications néonatales du fait de grossesses multiples ou de prématurité [46]. Il faut également souligner le coût élevé de chaque tentative en regard du taux de réussite ( 25 à $35 \%$ de grossesse par cycle) $[47,48]$.

\section{CONCLUSIONS}

Notre étude permet, du point de vue épidémiologique, de rendre compte du type de pathologie rencontrée dans une consultation ambulatoire. Elle montre qu'il est utile d'examiner l'homme infertile puisqu'on trouve des facteurs étiologiques de stérilité dans près de $80 \%$ des cas. Notre étude est critiquable du fait de son aspect rétrospectif et de l'absence de groupe de contrôle prédéfini. Elle permet pourtant de mettre en évidence que :

- le taux de grossesses spontanées est élevé, confirmant des travaux antérieurs (49);

- près d'un tiers des grossesses sont survenues à la suite de traitements andrologiques classiques, qui gardent toute leur valeur en cas d'altérations modérées du sperme (NTSM situé entre 5 et 15);
- en cas d'altérations plus sévères (NTMS 5) le recours à des techniques de PMA est nécessaire.

Il serait utile dans des études ultérieures d'analyser le rapport coût/efficacité des traitements andrologiques classiques comparés aux traitements recourant à la PMA en fonction des altérations du sperme et des facteurs de stérilité féminine [41, 43,48].

L'ICSI a permis de révolutionner le traitement de la stérilité masculine en faisant subir à la femme des traitements efficaces mais non dénués de risque. Ces progrès se font souvent aux dépens de l'investigation de recherche de causes spécifiques de stérilité masculine. Le traitement de cette dernière devrait être recentré sur l'homme infertile de façon à ce qu'il ne soit pas réduit au rôle de simple producteur de gamètes mais qu'il soit un partenaire à part entière dans le traitement de la stérilité du couple.

Remerciements : Nous remercions Madame Mermillod pour ses conseils avisés dans les analyses statistiques. Nous remercions tous les gynécologues qui ont participé à cette étude, en particulier les Drs B.Bourrit, P. Becker, L. Biéler, A. Bruckner, G. de Candolle, P. Dubouloz, R. Faigaux, A. Finci, E. Floris, J-F. Huber, M. Huber, C. Leuenberger, O. Nigg, C. Page, M. Pirek, A. Stalberg, D. Tonna, T. Tran-Ngoc, Y. Wespi, R. Wyss et D. de Ziegler ainsi que les Drs G. de Boccard, D. Chollet et J.Sauvain, urologues.

\section{REFERENCES}

1. SKaKkebaek N.E., Giwercman, A., DE KRETSER, D. : Pathogenesis and management of male infertility. Lancet $1994 ; 343: 1473-1478$.

2. Palermo G.D., Cohen J., Alikani M., Adler A., ROSENWAKS Z. : Intracytoplasmic sperm injection : a novel treatment for all forms of male factor infertility. Fertil Steril $1995 ; 63: 1231-1240$.

3. Nagy Z.P., LiU J., Joris H., Verheyen G., Tournaye H., Camus M., Derde M.-P., Devroey P. and VaN StEIRTEGHem A.C. : The result of intracytoplasmic sperm injection is not related to any of the three basic sperm parameters. Hum. Reprod. 1995a ; $10: 1123-1129$. 
4. Cummins J.M. AND Jequier A.M. : Treating male infertility needs more clinical andrology, not less. Hum Reprod 1994 ; 9 : 1214-1219.

5. BAKER H.W.G. : Male infertility. In : de Groot L.J. ed. Endocrinology, 3 rd ed. 1995 Orlando: Saunders p. 2405-2433.

6. Rowe P.J., Comhaire F.H., Hargrave T.B., Mellows H.J. : WHO Manual for the Standarized Investigation and diagnosis of the infertile Couple. Cambridge University Press, Cambridge, UK. 1993.

7. Soldati G., Piffaretti Yanez A., Campana A., MarCHINI M., LueRTI M. AND BALERNA M. : Effect of peritoneal fluid on sperm motility and velocity distribution using objective measurements. Fertil Steril $1989 ; 52: 113-119$.

8. World Health ORGanization : WHO Laboratory Manuel for the examination of human semen-cervical mucus interaction. 2nd. 1987. ed. Cambridge University Press, Cambridge.

9. Tournaye H., Liu J., Nagy Z., Camus M., Goossens A., Silber S., Van Steirteghem A., Devroey P. : Correlation between resticular histology and outcome after intracytoplasmic sperm injection using testicular spermatozoa. Hum Reprod $1996 ; 11$ : 127-132

10. Comhaire F.H., Rowe P.J. ANd Farley T.M.M. : The effect of doxycycline in infertile couples with male accessory gland infection : a double blind prospective study. Int $J$ Androl 1995; 9: 91-98.

11. Nieschlag E., Hertle L. and Behre H.M. : Treatment of varicocele; counselling as effective as occlusion of the vena spermatica. Hum. Reprod. $1995 ; 10: 347-353$.

12. ARNy M., Quagliarello J. : Semen quality before and after processing by a swim-up method : relationship to outcome of intrauterine insemination. Fertil Steril $1987 ; 48: 643-647$.

13. Martin-Du Pan R.C., Bourrit B., Biondo, M. : Traitement de la stérilité masculine par fécondation in vitro. Schweiz Med Wochenschr $1987 ; 117$ : 804-809.

14. Martin-Du Pan R.C., Sakkas D., Stalberg A., DE Boccard P.G., Campana A. : Traitement de la stérilité masculine par injection intra-ovocytaire de spermatozoide : évaluation critique. Schw. Med. Wochenschr 1995a ; $125: 1483-1488$.

15. Martin-Du Pan R.C., Bischof P., Campana A., Morabia A.: Relationship between etiological factors and total motile sperm count in 350 infertile patients.(soumis à Andrologia, 1997).

16. DUBIN L. AND AMELAR R.D. : Etiologic factors in 1,294 consecutive cases of male infertility. Fertil Steril $1971 ; 22: 496$.
17. NiEsChlaAg E.,BEHRE H.M. : Male infertility due to testicular dysfunction. In Drife,J.O. and Templeton,A.A. (eds).Infertility Procedings of the 25th Study Group of the Royal College Obstetricians and Gynecologists. Springer London 1992,pp. 65-80.

18. Barratt C.L.R.,Naeeni M.,Clements S., Cooke I.D. : Clinical value of sperm morphology for invivo fertility : comparison between World Health organization criteria of 1987 and 1992. Hum Reprod $1995 ; 10: \mathbf{5 8 7 - 5 9 3 .}$

19. Matorras R., Corcostegui B., Perez C., Mandolla M., Mendoza R., Rodriguez-Escudero F.J. : Sperm Morphology analysis (strict criteria) in male infertility is not a prognostic factor in intrauterine insimination with husband's sperm. Fertil Steril $1995 ; 63: 608-611$.

20. Ayala C., STEInBerger E., SMith D.P. : The impact of the male factor on fertility rates in infertile couples; an aralysis of large population. 20-th Annual Meeting for American Society of Andrology. J Androl. (Suppl. January-February 1995) Abstr. 24 : 9.

21. SILBER S.J. : The relationship of abnormal semen parameters to male fertility. Hum Reprod 1989 ;4 : 947-953.

22. BRasch J.G., RaWlins R., Tarchala S., RadWANSKa E. : The relationship between total motile sperm count and the success of intrauterine insemination. Fertil Steril $1994 ; 62: 150-154$.

23. Ben-Chetri A., Senoz S., Greendblatt E.M. AND CASPER R.F. : In vitro fertilization outcome in the presence of severe male factor infertilitiy. Fertil Steril $1995 ; 63$ : 1032-1037.

24. Ord T., Patrizio P., Balmaceda J.P., Asch R.H. : Can severe male factor infertility be treated without micromanipulation? Fertil Steril 1993 ; $60: 110-115$.

25. BAKER H.W.G. AND Kovacs G.T. : Spontaneous improvement in semen quality : regression toward the mean. Int J Androl $1985 ; 8: 421-426$.

26. WOLFF H. : The biologic significance of white blood cells in semen. Fertil Steril $1995 ; 63: 1143-1157$.

27. BRANIGAN E.F. AND MULLER C.H. : Efficacy of treatment and recurrence rate of leukycytospermia in infertile men with prostatitis. Fertil Steril 1994 ; $62: 580-584$.

28. Yanushrolsky E.H., Politch J.A., Hill J.A. AND ANDERSON D.J. : Antibiotic therapy and leukocytospermia : a prospective, randomized, controlled study. Fertil Steril $1995 ; 63: 142-147$.

29. SCHLESINGER M.H. AND HARRIS M. : Treatment outcome after varicocelectomy. Urol Clin North Am $1994 ; 21: 517$. 
30. MADGAR I., WeISSENBERG R., Lunenfeld B., KaraSIK A., GoLDWASSER B. : Controlled trial of high spermatic vein ligation for varicocele in infertile men. Fertil. Steril. 1995 ; $63: 120-123$.

31. O'Donovan P.A., VANDEKERCKHove P., LILFORD R.J. AND Hughes E. : Treatment of male infertility : is it effective ? Review and meta-analyses of published randomized controlled trials. Hum.Reprod. $1993 ; 8: 1209-1222$.

32. Moskovitz B., Lin R., Nassar S. AND Levin D.R. : Effect of Diclofenac Sodium (Voltaren) on Spermatogenesis of Infertile Oligospermic Patients. Eur.Urol. 1988 ; 14 : 395-397.

33. ScHOYSMAN R. : Epididymal causes of male infertility : pathogenesis and management. Int $\mathrm{J}$ Androl 1981 ; suppl. 5 : 120-133.

34. Hauser R., Temple-Smith P.D., Soudhwick G.J., McFarlane J. AND DE KRETSER D.M. : Pregnancies after microsurgical correction of partial epididymal and vasal obstruction. Hum Reprod $1995 ; 10$ : 152-1155.

35. Martin-Du Pan,R.C.,Bischof,P.,DE Boccard G.,Campana A. : Is diclofenac helpful in the diagnosis of partial epididymal obstruction? (sous prese, Human Reprod. 1997 ; 12).

36. Silber J.S., Nagy Z., Liu J., Tournaye H., Lissens W., Ferec C., Liebaers I., Devroey P. and Van STEIRTEGHem A.C. : The use of epididymal and testicular spermatozoa for intracytoplasmic sperm injection : the genetic complications for male infertility. Human Reprod $1995 ; 10$ : 2031-2043.

37. Campana A., Sakkas D., Stalberg A., Bianchi P.G., Comte I., PAChe T., Walker D. : Intrauterine insemination : evaluation of the results according to the woman's age, sperm quality, total sperm count per insemination and life table analysis. Hum Reprod $1996 ; 11$ : 732-736.

38. Kirbi C.A., Flaherty S.P., Godfrey B.M., Warnes G.M., MATTHEwS C.D. : A prospective trial of intrauterine insemination of motile spermatozoa versus timed intercourse. Fertil Steril $1991 ; 56$ : 102-107.

39. SilverberG K., Johnson J., Olive D., Burns W., SCHENKEN R.S. : A prospective,randomized trial comparing two different intrauterine insemination regimens in controlled ovarian hyperstimulation cycles. Fertil Steril $1992 ; 57: 357-361$.

40. Rosenberg S., Yuzpe A.A., Alken M., DunN R.C., Gantt P., Gianfortoni J.G., Maxson W.S.,WebsTER B. : A randomized comparative multicenter trial of ovulation induction (OI) using FSH/hCG vs. intrauterine insemination (IUI) vs. combined OI and IUI for couples with male factor infertility. The American Fertility Society. 1993 ; Montreal 11-14 oct. 1993.Abstract P-183.
41. Crosignani P.G.,Walters D.E. : Clinical pregnancy and male subfertility; the ESHRE multicentre trial on the treatment of male subfertility. Human Reprod $1994 ; 9$ : 1112-1118.

42. Hinting A., Comhaire F., Vermeulen L.,Dhont M., Vermeulen M., VANdekerckHove D. : Possibilities and limitations of techniques of assisted reproduction for the treatment of male infertility. Human Reprod $1990 ; 5: 544-548$.

43. SCHLEGEL P.N. : Is varicocelectomy a cost-effective treatment for male infertility in the ICSI era ? 51 st Meeting American Society of Reproductive Medecine 1995 ; 7-12 October, Seattle, USA. Abstr.0-177, pp 87.

44. Reijo R., Alagappan R.K., Patrizio P., Page D.C. : Severe oligozoospermia resulting from deletions of azoospermia factor gene on $\mathrm{Y}$ chromosome. Lancet $1996 ; 347: 1290-1293$.

45. SCHENKER J.G. AND EZRA Y. : Complications of assisted reproductive techniques. Fertil Steril $1994 ; 61$ : 415-418.

46. Callahan T.L., Janet J.E., Ettner S.L., Greene M.F., CrowleY W.F. : The economic impact of multiple-gestation pregnancies and the contribution of assisted-reproduction techniques. New Engl J Med $1994 ; 331$ : 244-249.

47. Neumann P.J., Gharib,S.D. and WeInstein M.C. : The Cost of a Successful delivery with in Vitro Fertilization. N Eng J Med 1994 ; 331 : 239-243.

48. Dor J., Seidman D.S., Ben-Shlomo I., Levran D., Ben-Rafael Z., Mashiach S. : Cumulative pregnancy rate following in-vitro fertilization : the significance of age and fertility aetiology. Hum Reprod $1996 ; 11: 424-428$.

49. Collins J.A.,Wrixon W., JANes L.B. AND Wilson E.H. : Treatment-independent pregnancy among infertile couples. N Engl J Med 1983 ; 309 : 12011206.

\section{ABSTRACT}

\section{Etiologic factors of male sterility evaluated in 350 men - efficiency of treatments}

\author{
R.C. Martin-du Pan, A. Campana
}

Etiologic factors of male sterility and the effect of different treatments on sperm quality have been evaluated in 350 men consulting for primary or secondary infertility of more than one year. Environmental factors represent 
$12 \%$ of the etiologies, acquired diseases such as varicoele and prostatitis $36.8 \%$, testicular failure $23 \%$, endocrinopathy $1,4 \%$, sexual dysfunction $1,4 \%$, abnormality of sperm transport $7,4 \%$. and idiopathic cases $19 \%$. A conventionnal treatment has been tempted in $50 \%$ of the cases and evaluated in 101 patients. A normalisation of the sperm (defined by a total motile sperm count (TMS) higher than 16 millions) has been observed in a variable proportion of the cases : $80 \%$ after hormonotherapy, $37 \%$ after varicocelectomy, and $30 \%$ after antiinflammatory treatment.
Among 100 pregnancies, $39 \%$ occurred either spontanously or after ovulation induction, $32 \%$ after classical andrological treatment and $26 \%$ after assisted reproductive techniques (ART) such as in vitro fertilization, intracytoplasmic sperm injection or intrauterine insemination. The efficiency of these different treatments according to the TMS and the utility of the clinical investigation of the infertile male are discussed. The risks faced by the wife because of ART are underlined.

Key-words : male sterility, drug treatment,assisted reproductive techniques, etiology. 